\title{
An unexpected absence of queuosine modification in the tRNAs of an Escherichia coli B strain
}

\author{
T. K. Dineshkumar, Swapna Thanedar, C. Subbulakshmi \\ and Umesh Varshney
}

Department of Microbiology and Cell Biology, Indian Institute of Science, Bangalore 560012, India
Author for correspondence: Umesh Varshney. Tel: +91 80394 2686. Fax: +91 803602697. e-mail:varshney@mcbl.iisc.ernet.in

The post-transcriptional processing of tRNAs decorates them with a number of modified bases important for their biological functions. Queuosine, found in the tRNAs with GUN anticodons (Asp, Asn, His, Tyr), is an extensively modified base whose biosynthetic pathway is still unclear. In this study, it was observed that the tRNA ${ }^{\text {Tyr }}$ from Escherichia coli B105 (a B strain) migrated faster than that from E. coli CA274 (a K-12 strain) on acid urea gels. The organization of tRNA $^{\text {Tyr }}$ genes in E. coli B105 was found to be typical of the B strains. Subsequent analysis of tRNA ${ }^{\text {Tyr }}$ and tRNA ${ }^{\text {His }}$ from several strains of $E$. coli on acid urea gels, and modified base analysis of tRNA preparations enriched for tRNA ${ }^{\text {Tyr }}$, showed that E. coli B105 lacked queuosine in its tRNAs. However, the lack of queuosine in tRNAs was not a common feature of all $E$. coli B strains. The tgt and queA genes in B105 were shown to be functional by their ability to complement tgt and queA mutant strains. These observations suggested a block at the step of the biosynthesis of preQ ${ }_{1}$ (or preQ $Q_{0}$ ) in the B105 strain. Interestingly, a multicopy vector harbouring a functional tgt gene was toxic to E. coli B105 but not to CA274. Also, in mixed cultures, E. coli B105 was readily competed out by the CA274 strain. The importance of these observations and this novel strain (E. coli B105) in unravelling the mechanism of preQ $\mathbf{~}_{1}$ or preQ $_{0}$ biosynthesis is discussed.

Keywords: tyrT-tyr $V$, tyr $U$, preQ $_{1}$, preQ ${ }_{0}$, acid urea gels

\section{INTRODUCTION}

Transfer RNAs contain a variety of modified nucleosides, of which at least 80 have been catalogued so far (McCloskey et al., 1998; Rozenski et al., 1999). One of the most elaborately modified nucleosides among these is queuosine (7-\{5-[(4S,5R-dihydrooxy-2-cyclopenten$1 S$-yl)amino]methyl $\}-7$-deazaguanosine). Introduction of queuosine into tRNAs is initiated by a transglycosylation reaction wherein a transglycosylase (Tgt) exchanges the guanine base at the first position of the GUN anticodon in tRNAs (Asp, Asn, His, Tyr) with 7aminomethyl-7-deazaguanine $\left(\mathrm{preQ}_{1}\right)$, a precursor of the base (queuine) of queuosine (Q). Subsequently a substituted cyclopentenyl group is attached to preQ $_{1}$ in tRNA by $S$-adenosylmethionine-tRNA ribosyltransferase/isomerase (QueA). The resultant epoxy-

Abbreviations: preQ $_{0}, 7$-cyano-7-deazaguanine; preQ $_{1}, 7$-aminomethyl7-deazaguanine. queuosine (oQ), 7-\{5-[(2,3-epoxy-4,5-dihydroxy-

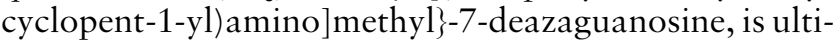
mately converted into queuosine by an as yet unrevealed enzyme that requires vitamin $B_{12}$. Besides $p$ reQ $Q_{1}$, Tgt can also use 7-cyano-7-deazaguanine $\left(\mathrm{preQ}_{0}\right)$, another deazaguanine compound, as substrate. In Escherichia coli, both QueA and Tgt are monomers, of 39 and $46 \mathrm{kDa}$, respectively, and are encoded from a single operon (Slany \& Kersten, 1994).

The queuosine in tRNAs has been proposed to modulate the interaction between the anticodon and the degenerate codons (Meier et al., 1985), and shown to be important for survival of $E$. coli in the natural environment (Noguchi et al., 1982). Interestingly, in Shigella flexneri, vacC, a virulence-associated chromosomal locus, is homologous to tgt of E. coli and a vacC mutant is complemented by tgt from E. coli (Durand et al., 1994). The mutations in vac C in S. flexneri result in the loss of pathogenicity, suggesting tgt as a possible drug target for shigellosis (Gradler et al., 2001). On the 
other hand, many tumours and neoplastic cell lines are deficient in queuosine modification (Aytac \& Gunduz, 1994; Harada \& Nishimura, 1972; Okada et al., 1979; Randerath et al., 1984). It has been suggested that queuosine modification in tRNA regulates protein synthesis and influences cellular growth and differentiation in tumour cells (Morris et al., 1999). The eukaryotes are unable to carry out de novo synthesis of queuine, and as a nutritional factor, queuine has also been suggested to modulate receptor tyrosine kinases (Langgut, 1995). As the eukaryotes obtain queuine through diet or the gut microflora (Slany \& Kersten, 1994), its analogues could serve as important therapeutic agents to treat cancer or related ailments (Nishimura, 1972; Nishimura et al., 1983).

Despite such crucial biological implications of queuosine modification in the tRNAs, the details of the biosynthesis of $\operatorname{pre}_{1}$ (or preQ $\mathrm{Q}_{0}$ ) base are largely unknown. Availability of well-characterized mutants is an important tool in exploring the complex biochemical steps in the biosynthetic pathways. During the course of our studies on structure-function relationship of initiator tRNA, we discovered that an isolate of E. coli (E. coli B105) lacked queuosine modification. Further characterization of this strain suggests that the lack of queuosine in tRNAs in this strain is due to some defect(s) at the step(s) prior to the transglycosylation step. Using this novel strain, it should now become possible to gain insights into the steps involved in the de novo biosynthesis of the precursors of the base of queuosine.

\section{METHODS}

Bacterial strains and growth media. The strains (Table 1) were cultured in Luria-Bertani (LB) broth $(1 \%$ tryptone, $0.5 \%$ yeast extract and $1 \%$ sodium chloride, w/v) or in solid LB (containing $1.5 \%$ agar). When needed, antibiotics were used at the following concentrations: ampicillin, $100 \mu \mathrm{g} \mathrm{ml}^{-1}$; and kanamycin, $25 \mu \mathrm{g} \mathrm{ml}^{-1}$ (Sambrook et al., 1989).
Isolation of tRNAs and Northern blot analysis. The tRNAs were isolated under cold and acidic conditions to preserve the ester linkage between the amino acid and tRNA, electrophoresed on $6.5 \%$ polyacrylamide gels containing $8 \mathrm{M}$ urea under acidic conditions (Varshney et al., 1991), electroblotted onto nytran membranes and hybridized (Thanedar et al., $2000)$ with $5^{\prime}-{ }^{32} \mathrm{P}$-end-labelled oligonucleotide. The probes were complementary to positions $29-47$ of tRNA ${ }^{\text {fMet(CUA) }}$, 2-37 of tRNA ${ }^{\text {Tyr }}$ or 30-49 of tRNA ${ }^{\text {His }}$ (Sprinzl et al., 1989). When needed, aminoacylated tRNAs were deacylated in $100 \mathrm{mM}$ Tris $/ \mathrm{HCl}(\mathrm{pH} \mathrm{9.0})$ at $37^{\circ} \mathrm{C}$ for $15 \mathrm{~min}$, recovered by ethanol precipitation and analysed on either acid urea or standard sequencing gels (Maxam \& Gilbert, 1980; Varshney et al., 1991).

Southern blot analysis. Genomic DNA $(5 \mu \mathrm{g})$ from E. coli B105 (a B-strain) and E. coli CA274 (a K-strain) were digested with BstEII or BstNI following the supplier's instructions, resolved on agarose gel $(0.9 \%)$, transferred onto nytran membrane under vacuum and hybridized with radiolabelled probe against tRNA ${ }^{\mathrm{Tyr}}$ (Reed \& Mann, 1985).

Cloning of the tyrU gene. The tyr $U$ locus was PCR amplified from both the E. coli strains, CA274 and B105. Genomic DNA (200 ng) and 40 pmol each of the forward (5'-GGTCACGCGTTCGATTCCGGTAG-3') and reverse (5'-ACGGATCCATCGGTGATATCACC-3') primers were used in PCR with Vent DNA polymerase (New England Biolabs). The reactions were subjected to initial incubation at $94^{\circ} \mathrm{C}$ for $5 \mathrm{~min}$ followed by 30 cycles each consisting of $1 \mathrm{~min}$ at $94^{\circ} \mathrm{C}, 1 \mathrm{~min}$ at $55^{\circ} \mathrm{C}$ and $1.5 \mathrm{~min}$ at $68^{\circ} \mathrm{C}$. The PCR products were used for cycle sequencing; the one from B105 was cloned into p Trc99C and referred to as pTrctyr $U$.

Cloning of queA and tgt genes and generation of an internal deletion in tgt ( $\Delta$ tgt). The que $A$ and $t g t$ cistrons from $E$. coli CA274 and E. coli B105 were PCR amplified using $200 \mathrm{ng}$ genomic DNA, $40 \mathrm{pmol}$ each of forward (5'-AGAATTCATCGATTATATTCTATCC-3') and reverse (5'-CGAATTCAAAGACATCGGGCTA-3') primers and $P f u$ DNA polymerase (Promega). After initial denaturation at $94{ }^{\circ} \mathrm{C}$ for $5 \mathrm{~min}$, DNAs were amplified in 30 cycles each consisting of $1 \mathrm{~min}$ at $94^{\circ} \mathrm{C}, 1 \mathrm{~min}$ at $45^{\circ} \mathrm{C}$ and $5 \mathrm{~min}$ at $68^{\circ} \mathrm{C}$. The $2.5 \mathrm{~kb}$ PCR products from B105 and CA274 were eluted from agarose gel, purified, digested with EcoRI and cloned into pTZN (a pTZ19R-derived vector) linearized with EcoRI, and referred

Table 1. Strains used in the study and their genotypes

\begin{tabular}{|c|c|c|}
\hline E. coli strain & Genotype & Reference \\
\hline CA274 & Hfr, lacZ125(Am) trpA49(Am) relA1 spoT & Brenner \& Beckwith (1965) \\
\hline CA275 & Hfr, lacZ125(Am) trpA49(Am) relA1 spoT1 tyrT66(As) & Brenner \& Beckwith (1965) \\
\hline B105 & An E. coli $\mathrm{B}$ strain $\mathrm{r}_{\mathrm{B}}^{-} \mathrm{m}_{\mathrm{B}}^{-} \mathrm{Gal}^{-} \mathrm{RecA}^{+}$ & Mandal \& RajBhandary (1992)* \\
\hline MRE600 & $\mathrm{F}^{-}, r n a$ & Gay (1984) \\
\hline MG1665 ( = DSM 3901) & A wild-type K-12 strain & Cayley et al.(1989) \\
\hline BL21(DE3) & $\mathrm{F}^{-}, h s d S_{\mathrm{B}}\left(\mathrm{r}_{\mathrm{B}}^{-} \mathrm{m}_{\mathrm{B}}^{-}\right) \operatorname{gal}(d c m) o m p T[l o n]$ & Studier \& Moffatt (1986) \\
\hline KL16 & relA1 spoT1 thi1 & Low $(1968)$ \\
\hline HB101 & $\begin{array}{l}\mathrm{F}^{-} \Delta(g p t-p r o A) 62 \text { leuB6 supE44 ara-14 } \Delta(m c r C-m r r) \text { rpsL20 xyl-5 } \\
\text { mtl-1 recA13 lacY1 galK2 }\end{array}$ & Messing (1981) \\
\hline JE7334 & lacY thi xyl metE ara & Noguchi et al. (1982) \\
\hline JE7336 & tgt lacZ xyl thi trp leu & Noguchi et al. (1982) \\
\hline JE10651 & queA & Reuter (1991) \\
\hline
\end{tabular}

*First cited reference of the E. coli B105 strain. 
to as pTZqueA-tgt(B105) and pTZqueA-tgt(CA274), respectively. Another construct, pTZqueA-tgt1(CA274), was similar to pTZqueA-tgt(CA274), except that it contained the queAtgt insert in the opposite orientation. pTZqueA-tgt(CA274) was digested with EcoRV to excise an internal segment ( $\sim 200$

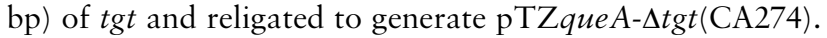

Modified base analysis. Exponentially growing cells of E. coli KL16 or B105 harbouring pTrctyrU(B105) were harvested and metabolically labelled with $\left[{ }^{32} \mathrm{P}\right]$ orthophosphate in low-phosphate medium (Seong \& RajBhandary, 1987; Thanedar et al., 2001). The total tRNAs were fractionated on $15 \%$ polyacrylamide gels under non-denaturing conditions. The region in the gel corresponding to tRNA ${ }^{\text {Tyr }}$ was localized with the help of ethidium bromide staining of the gel and the marker tRNA $^{\text {Tyr }}$ (a gift from Dr U. L. RajBhandary, Biology Department, Massachusetts Institute of Technology, Cambridge, USA), cut out and eluted in $5 \times$ TE containing $5 \%$ phenol and $1 \mathrm{M} \mathrm{LiCl}$. The tRNA preparation (enriched for $\mathrm{tRNA}^{\mathrm{Tyr}}$ ) was further purified by three steps of phenol extraction.

The labelled tRNA ${ }^{\mathrm{Tyr}}$ (25000 c.p.m.) was mixed with $1 \mu \mathrm{g}$ tRNA $^{\text {Tyr }}$ and digested with 1 unit RNase T2 in $20 \mathrm{mM}$ ammonium acetate buffer $(\mathrm{pH} 5.0)$ at $37^{\circ} \mathrm{C}$ for $5 \mathrm{~h}$, then subjected to repeated drying under vacuum to remove traces of ammonium acetate. The dried sample was resuspended in 3-4 $\mu \mathrm{l}$ water and spotted on cellulose sheets for twodimensional chromatography (Noguchi et al., 1982). The thinlayer chromatography was carried out using isobutyric acid/ $0.5 \mathrm{M}$ ammonia $5: 3(\mathrm{v} / \mathrm{v})$ solvent for the first dimension for 15-18 h until the solvent reached the top of the plate. The plates were dried for $12 \mathrm{~h}$ and then run in the perpendicular direction in the second solvent system (2-propanol/ water/HCl, 14:3:3, by vol.) (Nishimura, 1972), air-dried and subjected to autoradiography using a BioImage analyser (Fuji Film BAS1800).

Complementation studies. The constructs (pTZqueA-tgt of CA274 or B105 origin) or the vector control (pTZN) were transformed into $\operatorname{tg} t$ mutant (JE7336), $\operatorname{tg} t^{+}$(JE7334) or queA mutant (JE10651) strains. The tRNA preparations were analysed by Northern blotting.

Growth competition experiments. The cultures of E. coli CA274 and B105 were started with $0.25 \%$ inoculum from overnight cultures in $25 \mathrm{ml} \mathrm{LB}$ broth and grown at $37^{\circ} \mathrm{C}$ with shaking. Samples were taken periodically to read $\mathrm{OD}_{600}$. In the growth competition assays, overnight cultures of E. coli CA274 and B105 were mixed in equal volumes (zero subculture) for use as inoculum and grown to saturation (first subculture). Inoculum from the first subculture was used to obtain the second subculture; and an inoculum from the second subculture yielded the third subculture. The abundance of CA274 and B105 in the cultures was determined by dilution plating on MacConkey's agar. E. coli CA274 with an amber mutation in the lac Z gene does not ferment lactose in the medium and produces white colonies, whereas E. coli B105, which utilizes lactose, grows as pink colonies.

\section{RESULTS}

\section{The tRNA ${ }^{\text {Tyr }}$ from $E$. coli B105 migrates faster than that from E. coli CA274 on high-resolution polyacrylamide urea gels}

Use of acid urea gels (Varshney et al., 1991) in our studies on the structure-function relationship of initiator tRNAs revealed that the Tyr-tRNA ${ }^{\text {Tyr }}$ from $E$. coli B105, a B strain, migrated faster than that from $E$. coli CA274, a K strain (Fig. 1a). In contrast, the aminoacyl- and formylaminoacyl- forms of the initiator tRNA from the two strains co-migrated (compare the fGln- and Gln-tRNA ${ }_{2}{ }^{\mathrm{fMet}(\mathrm{CUA})}$ bands in lanes 1-4 with lanes 5-8). Although a detailed description of the various forms of $\mathrm{tRNA}_{2}{ }^{\text {fMet(CUA) }}$ mutants is not justified here, they serve as a control to rule out deacylation of the tRNA preparations from the B105 strain. To further examine this mobility difference, the total tRNAs (deacylated) from E. coli CA274 and B105 were analysed by urea-PAGE under completely denaturing conditions (Fig. 1b: compare lanes 1 and 2 with lanes 3 and 4 ). Consistent with the observation in Fig. 1(a), the tRNA ${ }^{\text {Tyr }}$ from strain B105 migrated faster than that from CA274. We reasoned that this mobility difference could be due either to a very small insertion/deletion in $\mathrm{tRNA}^{\mathrm{Tyr}}$, or to differential post-transcriptional processing in the two strains.

\section{Organization of tRNA ${ }^{\mathrm{Tyr}}$ genes, and sequence analysis of tyrU from E. coli B105 and CA274}

In E. coli, $\mathrm{tRNA}^{\mathrm{Tyr}}$ is encoded by the tyrT-tyrV $(27 \mathrm{~min})$ and $\operatorname{tyr} \mathrm{U}(79 \mathrm{~min}$ ) loci (Fig. 2a). The tyrT-tyrV locus is transcribed from a single promoter, and in E. coli $\mathrm{K}$ strains this locus contains a $3.14 \times$ repeat of a $178 \mathrm{bp}$ element, downstream of the tyrV gene (panel i). Interestingly, in the B strains, there is a deletion of $357 \mathrm{bp}$ in the repeat region (panel ii) (Timms \& Bridges, 1996). These differences in the $K$ and $B$ strains warranted a study of the organization of tyrosine tRNA genes in the CA274 and B105 strains by Southern blot analysis using two diagnostic restriction endonucleases, BstEII and BstNI (Fig. 2b). The bands of $2 \cdot 2 \mathrm{~kb}$ in the BstEII digest (lanes 1 and 2) and $0.5 \mathrm{~kb}$ in the Bst NI digest (lanes 3 and 4) show that the position of the tyrU locus is conserved in both the strains (Fig. 2a, panel iii). The bands of $\sim 1.4$ and $1 \mathrm{~kb}$ seen in the BstEII digest (lanes 1 and 2) are also consistent with the expected pattern for the tyrT-tyrV genes of the $\mathrm{K}$ and $\mathrm{B}$ strains, respectively. The bands of $\sim 0.9,0.68$ and $0.5 \mathrm{~kb}$ in the Bst $\mathrm{NI}$ digest (lane 3) agree with the sizes of the tyrV, tyrT and tyrU genes in the $\mathrm{K}$ strain. Interestingly, for the B105 strain, while the sizes of the 0.68 and $0.5 \mathrm{~kb}$ bands for the tyrT and $\operatorname{tyr} U$, respectively, were conserved, the tyrV $(\sim 0.56 \mathrm{~kb}$, lane 4$)$ showed a deletion of $\sim 0.35 \mathrm{~kb}$ from the $0.9 \mathrm{~kb}$ band (compare tyrV bands in lanes 3 and 4 ). Taken together, these observations reveal that the overall organization of tRNA ${ }^{\text {Tyr }}$ genes in E. coli CA274 and B105 is typical of the $\mathrm{K}$ and $\mathrm{B}$ strains, respectively.

The deletion in the $3.14 \times$ repeat downstream of $t y r V$ in E. coli $\mathrm{B}$ has been proposed to be responsible for the instability of the nascent transcripts arising from this locus (Timms \& Bridges, 1996). Hence, in E. coli B, most of the tRNA ${ }^{\mathrm{Tyr}}$ originates from the tyr $U$ gene. Therefore, for a detailed analysis, we PCR amplified the tyr $U$ genes from both the strains and sequenced them. The sequences of $t y r U$ from both the strains were identical to each other and to the reported sequence of $t y r U$ (NCBI accession number X04181; GenBank accession number gi:43228), ruling out mutations in the tDNA as a 
(a)

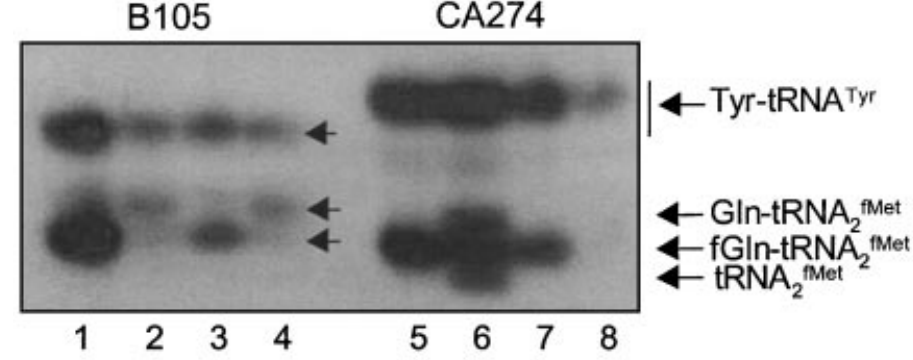

(b)

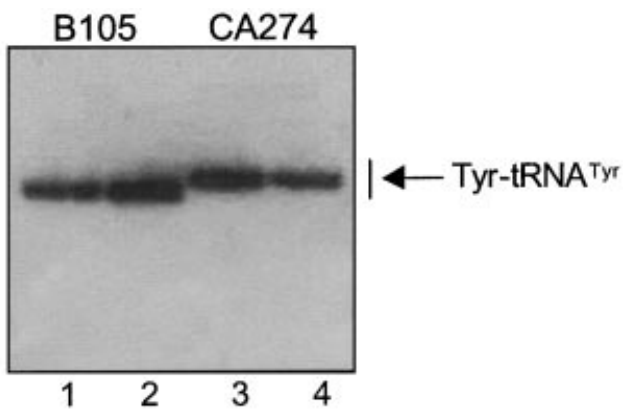

Fig. 1. Differential migration of Tyr-tRNA ${ }^{T y r}$ from $E$. coli $B 105$ and $C A 274$ strains on high-resolution gels. Total tRNA was prepared from E. coli B105 and CA274 harbouring mutant initiator tRNA (U35A36) genes under cold and acidic conditions and analysed on $6.5 \%$ acid urea gel (a) or a standard sequencing gel after deacylation (b), transferred to nytran

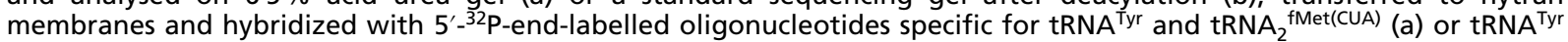
alone (b). Autoradiograms are shown. Lanes 1-4 in (a), and 1 and 2 in (b), represent samples from E. coli B105; lanes 5-8 in (a), and 3 and 4 in (b), represent samples from E. coli CA274. (Note: because of the U35A36 mutation, the initiator tRNAs are aminoacylated with glutamine.)

(a)

(i)

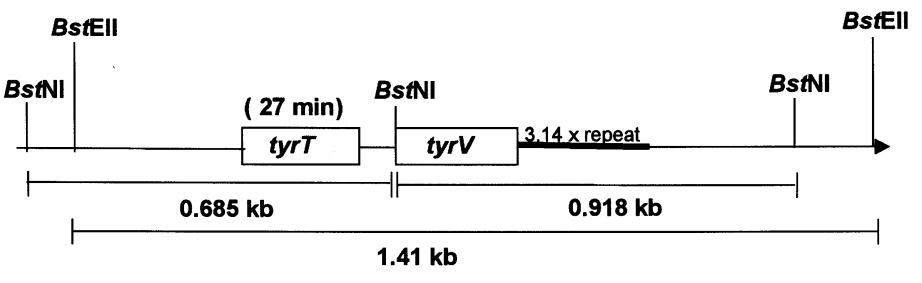

(ii)

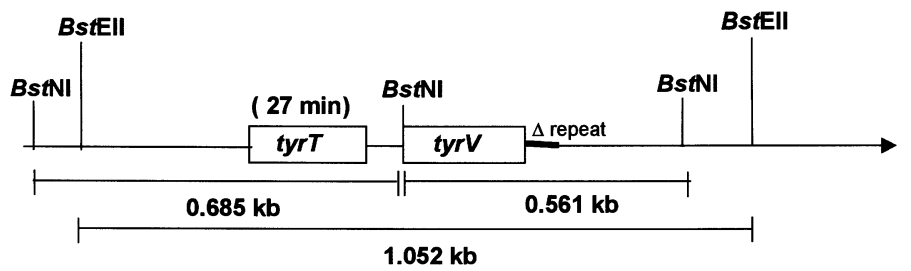

(iii)

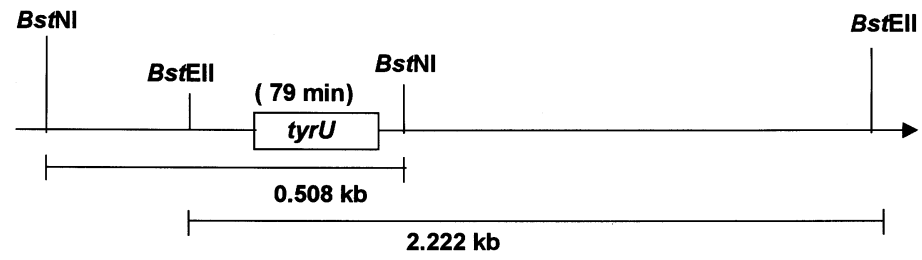

(b)

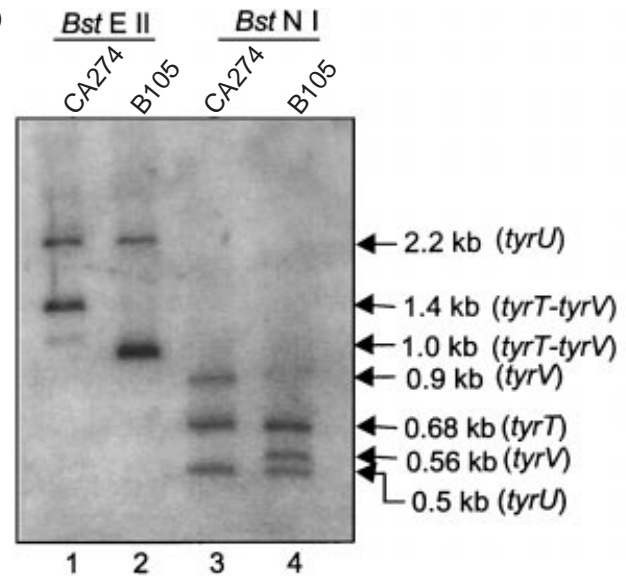

(c)

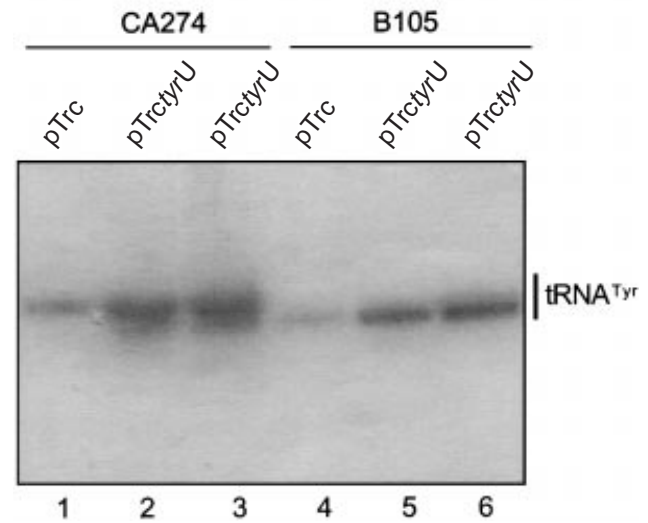

Fig. 2. (a) A schematic representation of the tyrosine tRNA genes in $E$. coli. The tyrT locus contains tandemly duplicated genes tyrT-tyrV followed by a $3.14 \times$ repeat element of $178 \mathrm{bp}$ in the $\mathrm{K}$ strains (panel i). In E. coli B strains (panel ii) this repeat element suffers a deletion of $357 \mathrm{bp}$ (Timms \& Bridges, 1996). The tyrU locus (panel iii) is conserved in both the strains. Sizes of the expected fragments upon digestion with BstEll and BstNI are as shown. (b) Southern blot analysis: Genomic DNA from E. coli B105 and CA274 was digested with BstEll and BstNI, resolved on 0.9\% agarose gel, transferred to nytran membrane and hybridized to ${ }^{32} \mathrm{P}$-labelled probe against tRNA ${ }^{\mathrm{Tyr}}$. An autoradiogram is shown. Sizes and the identities of various hybridizing DNA fragments are indicated on the right. (c) Overexpression of tyrU in E. coli B105 and CA274. Total tRNA from the transformants of E. coli CA274 (lanes 1-3) or B105 (lanes 4-6) harbouring either pTrc99C vector alone or pTrctyrU as indicated was fractionated on a sequencing gel and processed for Northern blot analysis using a ${ }^{32}$ P-labelled tRNA ${ }^{\mathrm{Tyr}}$-specific probe. Lanes 2 and 3, and lanes 5 and 6 , represent independent clones transformed with pTrctyru. 
(a)

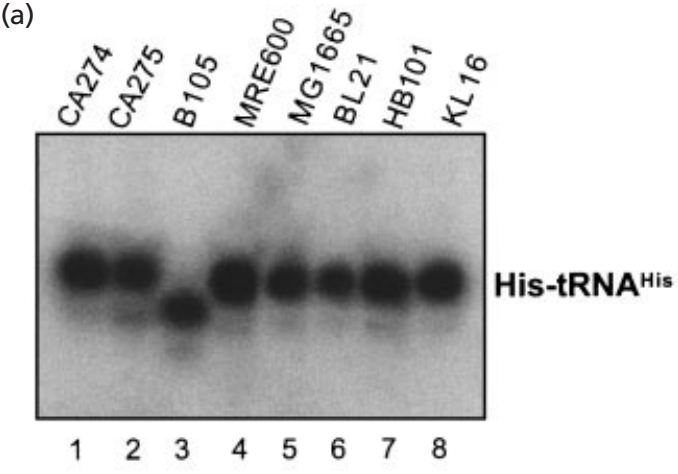

(b)

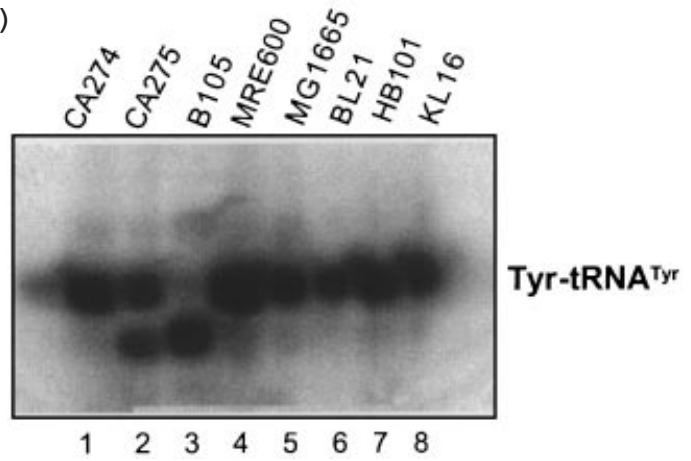

Fig. 3. Northern analysis for $t R N A^{\text {His }}$ and $t R N A^{\text {Tyr }}$. Total tRNA from various $E$. coli strains was prepared under cold and acidic conditions, fractionated on acid urea gels, transferred to nytran membranes and hybridized to ${ }^{32} \mathrm{P}$-labelled probes against tRNA $^{\text {His }}(\mathrm{a})$ or tRNA ${ }^{\text {Tyr }}(\mathrm{b})$.

possible reason for the mobility difference in $\mathrm{tRNA}^{\mathrm{Tyr}}$ of the two strains.

\section{Expression of the cloned tyrU gene in E. coli CA274 and B105}

To gain further insights, we transformed pTrctyr $U$ into E. coli CA274 and B105. The analysis of tRNAs showed that the introduction of $t y r U$ gene on a plasmid resulted in overproduction of $\mathrm{tRNA}^{\mathrm{Tyr}}$ in both the strains (Fig. 2c: compare lane 1 with 2 and 3 , and lane 4 with 5 and 6). Interestingly, the bulk of the tRNA ${ }^{\mathrm{Tyr}}$ from the CA274 strain still migrated slower than that from the B105 strain (compare lanes 2 and 3 with 5 and 6). Also, overproduction of $\mathrm{tRNA}^{\mathrm{Tyr}}$ in CA274 resulted in a faint band just below the major band (lanes 2 and 3), which co-migrated with the tRNA ${ }^{\mathrm{Tyr}}$ of the B105 strain. This is most likely due to lack of modification in a minor population of tRNA ${ }^{\mathrm{Tyr}}$ under these conditions. Taken together, these observations strongly suggest that the mobility differences in $\mathrm{tRNA}^{\mathrm{Tyr}}$ are not caused by mutation, and in all probability arise from strain-specific modifications.

\section{Lack of queuosine modification in E. coli B105}

Since the initiator tRNAs from the two strains comigrated on the same gel (Fig. 1a), differential biosynthesis of 2'-O-methylcytidine $(\mathrm{Cm})$, pseudouridine
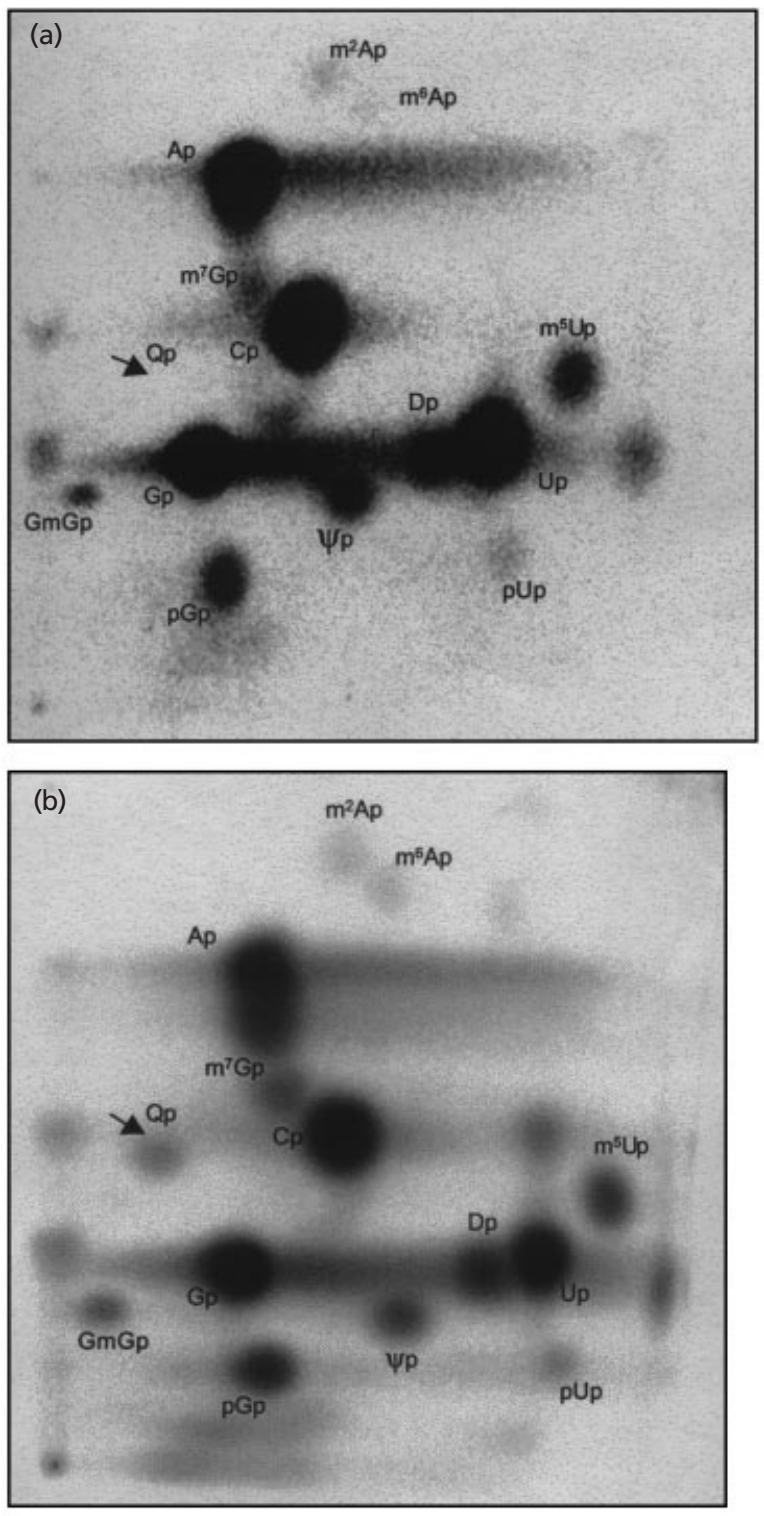

Fig. 4. Modified base analysis of tRNA ${ }^{\mathrm{Tyr}}$. The in vivo ${ }^{32} \mathrm{P}$-bodylabelled tRNA from E. coli B105 (a) or KL12 (CA274) (b) strains was enriched for tRNA ${ }^{T y r}$, digested with RNase T2, analysed by two-dimensional chromatography and autoradiographed. The presence or absence of a spot corresponding to queueosine $(\mathrm{Qp})$ is indicated by an arrow.

$(\psi)$, dihydrouridine (D), thiouridine $(\mathrm{S})$ and ribothymidine $(\mathrm{rT})$, which are common to the tRNA ${ }^{\mathrm{Tyr}}$ and the initiators (as well as other tRNAs) was unlikely to be responsible for the faster mobility of tRNA ${ }^{\text {Tyr }}$ from $E$. coli B105. We therefore focused our attention on queuosine modification, which is present in a set of tRNAs containing the GUN anticodon (encoding Tyr, His, Asp and Asn). The total tRNAs from several E. coli strains were fractionated on acid urea gels and probed for tRNA ${ }^{\text {His }}$ and tRNA ${ }^{\text {Tyr }}$ (Fig. 3a and b, respectively). Importantly, in this analysis we included E. coli CA275, which differs from the CA274 strain in that its tyr $T$ locus 
(a)

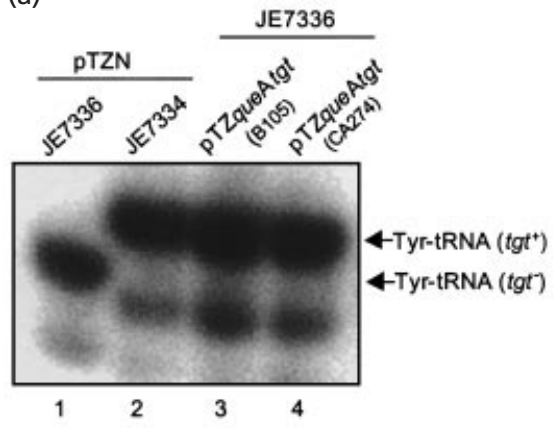

(b)

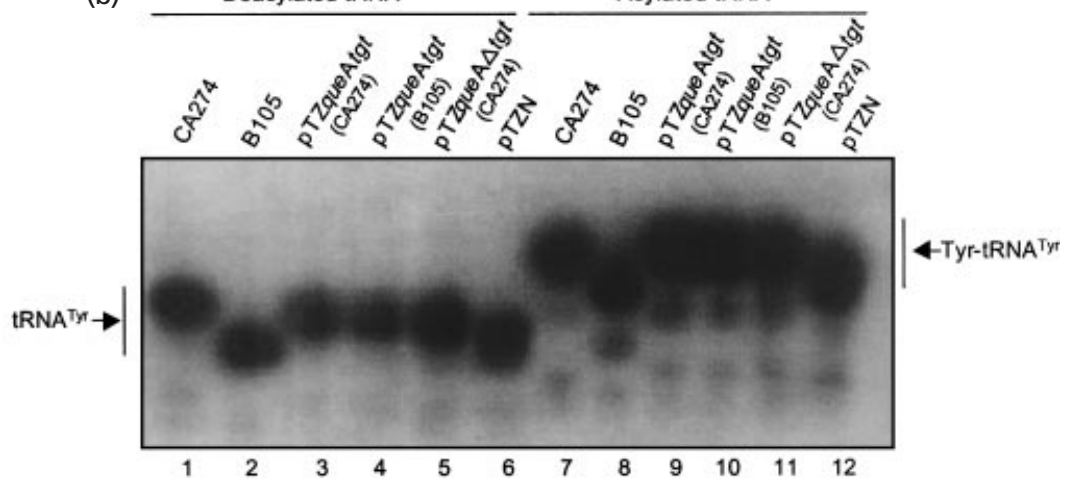

Fig. 5. (a) Complementation analysis using E. coli JE7334 (tgt $\left.t^{+}\right)$or JE7336 (tgt) strains. The vector pTZN (lanes 1 and 2 ) or the constructs pTZqueA-tgt(B105) (lane 3) or pTZqueA-tgt(CA274) (lane 4) were transformed into E. coli JE7334 (lane 2) or JE7336 (lanes 1, 3 and 4). Total tRNA was prepared under cold and acidic conditions, fractionated on acid urea gel, transferred to nytran membranes and hybridized to ${ }^{32}$ P-labelled probe against tRNA ${ }^{\text {Tyr }}$. (b) Complementation analysis using E. coli JE10651 (queA). The deacylated (lanes 1-6) or acylated (lanes 7-12) total tRNA samples from various transformants were fractionated on acid urea gel and processed as in (a). An autoradiogram is shown. Lanes: 1 and 7, total tRNA from $E$. coli CA274 harbouring PTZN vector; 2 and 8, total tRNA from E. coli B105 harbouring pTZN vector; 3-6 and 9-12, total tRNA from E. coli JE10651 harbouring plasmids as indicated on top of the lanes.

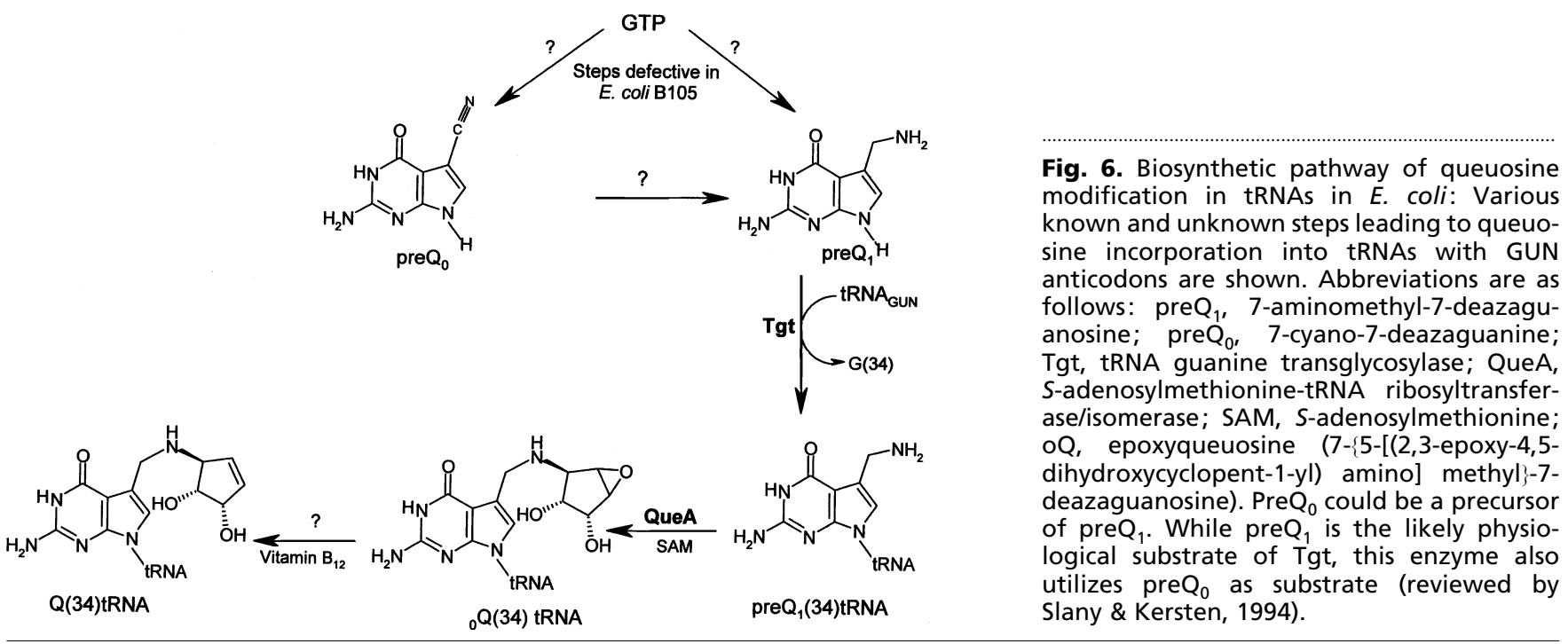

encodes a tRNA with CUA anticodon (an amber suppressor tRNA, supF).

Consistent with the prediction of the lack of queuosine modification, even the tRNA ${ }^{\text {His }}$ from E. coli B105 (Fig. 3a, lane 3) showed faster mobility. All other strains, including CA275, showed a single tRNA ${ }^{\text {His }}$ band comigrating with that from the CA274 strain. Remarkably, when the blot was probed for tRNA ${ }^{\mathrm{Tyr}}$, the E. coli strain CA275 (Fig. 3b, lane 2) showed two bands, the lower of which co-migrated with the band from $E$. coli B105. As in the CA275 strain, tyrT encodes tRNA ${ }^{\mathrm{Tyr}}$ $(s u p F)$, which cannot be modified at position 34 with queuosine (CUA anticodon); this observation was quite revealing and conveniently limited our investigation to the lack of queuosine modification in the B105 strain. Interestingly, it may be noted that the faster mobility of the tRNAs for His and Tyr was not seen for other E. coli
B strains such as BL21 and HB101, suggesting that the lack of the suspected (queuosine) modification was restricted to the B105 strain.

\section{Modified base analysis}

To confirm that the faster mobility of the His- and Tyrspecific tRNAs in the B105 strain was due to the lack/deficiency of queuosine modification, we carried out a modified base analysis (Noguchi et al., 1982) of tRNA preparations enriched for $\mathrm{tRNA}^{\mathrm{Tyr}}$ (Fig. 4). While a spot corresponding to queuosine $(\mathrm{Qp})$ was seen in the control $\mathrm{K}$ strain (Fig. 4b), it was absent from the B105 strain even upon overexposure of the autoradiogram (Fig. 4a), confirming that the B105 strain lacked queuosine modification. However, other identifiable modified nucleosides were present commonly in both the strains. 
Table 2. Number of transformants obtained upon introduction of various plasmids into $E$. coli CA274 and B105

\begin{tabular}{|lcc|}
\hline Construct & \multicolumn{2}{c|}{ No. of transformants obtained } \\
\cline { 2 - 3 } & E. coli CA274 & E. coli B105 \\
\hline Vector alone (pTZN) & 1116 & 796 \\
pTZqueA-tgt(CA274) & 792 & 0 \\
pTZqueA-tgt1(CA274) & 576 & 0 \\
pTZqueA- $\operatorname{tgt}(\mathrm{CA} 274)$ & 848 & 564 \\
pTZqueA-tgt(B105) & 476 & 0 \\
\hline
\end{tabular}

\section{The queA and tgt genes from E. coli B105 code for functional proteins}

To investigate the reasons for the lack of queuosine modification in the B105 strain, we cloned the tgt and queA genes from both the B105 and CA274 strains. The two constructs and the vector alone were transformed into JE7336 (tgt), JE7334 $\left(\operatorname{tg} t^{+}\right)$and JE10651 (queA) strains. As expected, the Northern analysis showed that the Tyr-tRNA ${ }^{\text {Tyr }}$ from JE7336 (tgt) harbouring vector alone migrated faster than that from JE7334 $\left(\right.$ tgt $\left.^{+}\right)$(Fig. $5 \mathrm{a}$ : compare lane 1 with lane 2). However, introduction of pTZqueA-tgt constructs (of either B105 or CA274 origin) into JE7336 restored the slow mobility to the Tyr-tRNA $^{\text {Tyr }}$ (compare lane 1 with lanes 3 and 4), suggesting that the tgt genes of the B105 and the CA274 strains encoded functional proteins. Similarly, while the difference in the mobility of the $\mathrm{tRNA}^{\mathrm{Tyr}}$ prepared from the queA mutant (JE10651) and the wild-type strain (JE7334) is not as prominent as for the $t g t^{+}$versus tgt mutant strains (JE7334 vs JE7336), there is a small mobility difference, irrespective of the acylation status of the samples (Fig. 5b: compare lanes 1 and 2 with 6, and 7 and 8 with 12). Interestingly, the mobility of tRNA ${ }^{\text {Tyr }}$ isolated from JE10651 harbouring pTZqueA$\operatorname{tg} t(\mathrm{~B} 105)$ was identical to that of the tRNA ${ }^{\mathrm{Tyr}}$ from CA274 (compare lane 4 with lane 1, and lane 10 with 7) and from JE10651 harbouring either queA-tgt or queA$\Delta$ tgt from CA274 (compare lane 4 with 3 and 5, and lane 10 with 9 and 11), indicating that the queA gene of B105 also encodes a functional protein. Since for queuosine modification in tRNA, the QueA step occurs after the Tgt step (Fig. 6), these data suggest that the defect in queuosine biosynthesis in the B105 strain must precede the Tgt step. Thus, the defect in B105 is most likely in the biosynthesis of $\mathrm{preQ}_{1}$ (or $\mathrm{preQ}_{0}$ ).

\section{Overproduction of Tgt in E. coli B105 is toxic}

To further investigate the consequences of the lack of Qbase modification, we wished to introduce the pTZqueA-tgt constructs into the B105 strain. As is clear from the number of transformants obtained in various experiments (Table 2), introduction of plasmids carrying queA-tgt genes, irrespective of their source [pTZqueA-
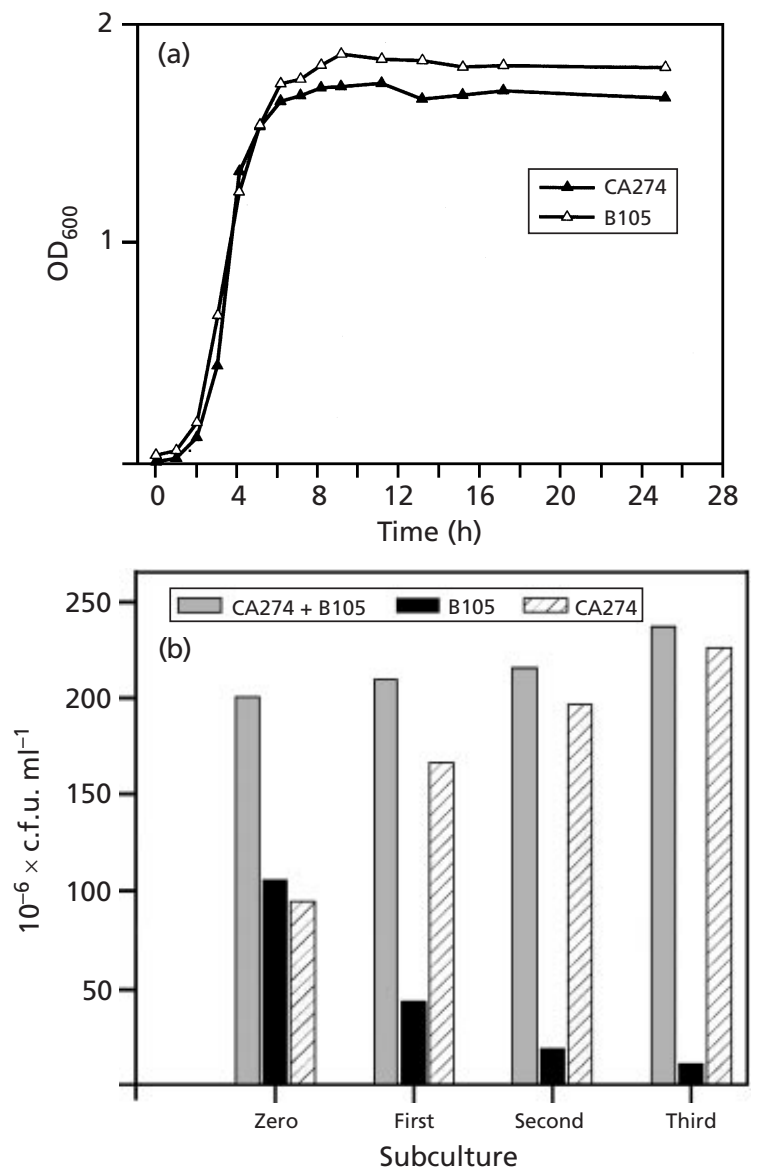

Fig. 7. (a) Growth curves of E. coli CA274 and B105 in LB medium. (b) Growth competition assay using mixed inoculum of E. coli CA274 and B105. Total viable counts corresponding to E. coli B105 and CA274 were determined by dilution plating on MacConkey's agar. The viable counts of the strains individually and in total are shown in the histogram. For details see Methods.

$\operatorname{tgt}(\mathrm{B} 105)$ or pTZqueA-tgt(CA274)] or orientation [pTZqueA-tgt(CA274) or pTZqueA-tgt1(CA274)], into E. coli was toxic to the B105 strain, but not to the CA274 strain. However, when we used a construct (pTZqueA$\Delta t g t)$ containing an internal deletion in the tgt gene, the construct was no longer toxic to the B105 strain (Table 2 ), suggesting that expression of Tgt from a multicopy vector is toxic to the B105 strain.

\section{Growth curve and competition studies}

Since the lack of queuosine in tRNAs in strain B105 was unexpected, we wished to study the fate of E. coli B105 in mixed-culture growth experiments (see Methods). The growth curves of the individually grown strains were very similar (Fig. 7a). However, when we carried out sequential subculturing from a mixed inoculum, we noticed that even though the mixed cultures were started with a slight advantage to B105 (as seen from the higher total viable counts for B105 in the inoculum for the zero 
subculture, Fig. 7b), this strain rapidly declined in favour of CA274 in the subsequent subcultures. While it remains to be established that the lack of queuosine modification in B105 contributes to its lesser fitness in this experiment, the observation is consistent with the reports that E. coli strains lacking queuosine in their tRNAs do not compete well with their counterparts containing this modification (Noguchi et al., 1982; Frey et al., 1989).

\section{DISCUSSION}

In this study, we show that E. coli B105 lacks queuosine modification in its tRNA. Our observation may appear in conflict with an earlier report on the presence of an intact pathway for queuosine modification in E. coli B (Harada \& Nishimura, 1972). However, it may be noted that the lack of queuosine modification was restricted to the B105 strain, and not a common feature of all B strains (Fig. 3). Furthermore, as demonstrated by the various complementation analyses using the tgt and queA mutants of E. coli, both the tgt and queA genes in the B105 strain encode functional proteins. These analyses have also shown that the lack of queuosine modification is most likely due to some $\operatorname{defect}(\mathrm{s})$ at step(s) prior to the Tgt step. In fact, the steps leading to biosynthesis of the precursors of the base of queuosine utilized as substrate by Tgt are entirely unknown. We believe that the availability of the B105 strain provides an important genetic system towards identifying the enzymic activities involved in these steps. Interestingly, expression of Tgt in E. coli B105 from a multicopy vector was found to be toxic. A possible explanation for this observation could be that Tgt cleaves the $\mathrm{N}$ glycosidic bond between the G34 and the ribose sugar of the tRNAs with GUN anticodons, leading to depurination. In the absence of $\mathrm{preQ}_{1}$ or $\mathrm{preQ}_{0}$, the covalent complex formed between the tRNA and Tgt (Romier et al., 1996) may sequester a significant amount of the substrate tRNAs, especially the low-abundance ones, and make them unavailable for protein synthesis.

The biosynthesis of $\mathrm{preQ}_{1}$ or $\mathrm{preQ}_{0}$ in bacteria is thought to result from the action of GTP-cyclohydrolase-like enzyme on GTP (Fig. 6). However, the two enzymes presently known to possess such activity (cyclohydrolase I and cyclohydrolase II) in E. coli are involved in pteridine and riboflavin biosyntheses, respectively, and their role in the biosynthesis of $\mathrm{preQ}_{1}$ or $\mathrm{preQ}_{0}$ has been ruled out. Moreover, a mutant of E. coli (ribA) defective in cyclohydrolase II still had queuosine modification in its tRNA (Slany \& Kersten, 1994). In fact, the only mutations that have been characterized so far in the pathway leading to queuosine modification in tRNA are at the steps of Tgt and QueA (Noguchi et al., 1982; Reuter et al., 1991). The E. coli B105 strain thus provides a tool to investigate the very early steps of biosynthesis of the precursor(s) of queuosine base. Importantly, since synthesis of the queuosine base is unique to prokaryotes (Slany \& Kersten, 1994), detailed knowledge of the enzyme(s) involved at this step could make it a novel target to design a new class of antibacterials.
Tgt overproduction is tolerated in E. coli CA274 but not in E. coli B105. The lethal phenotype in B105 may allow one to isolate gene(s) involved in the synthesis of the precursors of the base of queuosine by suppression of the toxic phenotype of Tgt overexpression. The result of the growth competition experiment shown in Fig. 7(b), which was performed to illustrate the reduced fitness of strain B105 due to lack of queuosine modification, would be more valid if carried out with isogenic strains. Nevertheless, our observation that B105 $\left(\mathrm{Q}^{-}\right)$fails to compete with CA274 $\left(\mathrm{Q}^{+}\right)$suggests that introduction of gene(s) from a library $\left(\mathrm{Q}^{+}\right)$into $\mathrm{B} 105$, followed by multiple subculturing, may result in enrichment/ selection of transformants wherein the resident plasmid confers a growth advantage to the host due to queuosine modification. Thus, characterization of the E. coli B105 strain in this study will be helpful in the elucidation of the queuosine biosynthetic pathway, which may even be a boon to the field of cancer biology (Nishimura et al., 1983) and contribute to a better understanding of the virulence of Shigella (Gradler et al., 2001).

Finally, the electrophoretic analysis of tRNAs on acid urea gels has provided a very useful and sensitive tool to separate the various forms (deacylated, aminoacylated or formylaminoacylated) of tRNA in a large number of studies. These gels have also provided crucial insights into the aminoacylation of tRNA with different amino acids (Li et al., 1996) or their differential modification status for the 2-methylthio- $N^{6}-\left(\Delta^{2}\right.$-isopentenyl)adenosine $\left(\mathrm{ms}^{2} \mathrm{i}^{6} \mathrm{~A}\right)$ (Mangroo et al., 1995). In this study, we provide yet another example of discrimination of tRNA species differentially modified for the queuosine base. In fact, detection of mobility differences even for the tRNAs isolated from the queA mutant background suggests that the acid-urea-gel-based separation system can be exploited for wide-ranging applications.

\section{ACKNOWLEDGEMENTS}

We thank the Japanese E. coli Stock Centre for providing us with the tgt and queA strains; and our laboratory colleagues for their suggestions on the manuscript. This work was supported by research grants from the Department of Science and Technology, and the Department of Biotechnology, New Delhi. S. T. was a Dr K. S. Krishnan senior fellow and T.K.D. was supported by a post-doctoral fellowship of the Department of Biotechnology.

\section{REFERENCES}

Aytac, U. \& Gunduz, U. (1994). Q-modification of tRNAs in human brain tumors. Cancer Biochem Biophys 14, 93-98.

Brenner, S. \& Beckwith, J. R. (1965). Ochre mutants, a class of suppressible nonsense mutants. J Mol Biol 13, 629-637.

Cayley, S., Record, M. T., Jr \& Lewis, B. A. (1989). Accumulation of 3-(N-morpholino)propanesulfonate by osmotically stressed Escherichia coli K-12. J Bacteriol 171, 3597-3602.

Durand, J. M., Okada, N., Tobe, T. \& 7 other authors (1994). vacC, a virulence-associated chromosomal locus of Shigella flexneri, is homologous to tgt, a gene encoding tRNA-guanine transglycosylase (Tgt) of E. coli K-12. J Bacteriol 176, 4627-4634. 
Frey, B., Janel, G., Michelsen, U. \& Kersten, H. (1989). Mutations in the Escherichia coli fnr and tgt genes: control of molybdate reductase activity and the cytochrome $d$ complex by $f n r$. J Bacteriol 171, 1524-1530.

Gay, N. J. (1984). Construction and characterization of an Escherichia coli strain with uncI mutation. J Bacteriol 158, 820-825.

Gradler, U., Gerber, H. D., Goodenough-Lashua, D. M., Garcia, G. A., Ficner, R., Reuter, K., Stubbs, M. T. \& Klebe, G. (2001). A new target for shigellosis: rational design and crystallographic studies of inhibitors of tRNA-guanine transglycosylase. J Mol Biol 306, 455-467.

Harada, F. \& Nishimura, S. (1972). Possible anticodon sequences of $\mathrm{tRNA}^{\mathrm{His}}$, tRNA ${ }^{\mathrm{Asn}}$, and $\mathrm{tRNA}^{\mathrm{Asp}}$ from Escherichia coli B. Universal presence of nucleoside $\mathrm{Q}$ in the first position of the anticodons of these transfer ribonucleic acids. Biochemistry 11, 301-308.

Langgut, W. (1995). Regulation of signaling by receptor tyrosine kinases in HeLa cells involves the q-base. Biochem Biophys Res Commun 207, 306-311.

Li, S., Kumar, N. V., Varshney, U. \& RajBhandary, U. L. (1996). Important role of the amino acid attached to tRNA in formylation and in initiation of protein synthesis in Escherichia coli. J Biol Chem 271, 1022-1028.

Low, K. B. (1968). Formation of merodiploids in matings with a class of $\mathrm{Rec}^{-}$recipient strains of Escherichia coli K12. Proc Natl Acad Sci U S A 60, 160-167.

Mandal, N. \& RajBhandary, U. L. (1992). Escherichia coli B lacks one of the two initiator tRNA species present in E. coli K-12. $J$ Bacteriol 174, 7827-7830.

Mangroo, D., Limbach, P. A., McCloskey, J. A. \& RajBhandary, U. L. (1995). An anticodon sequence mutant of Escherichia coli initiator tRNA: possible importance of a newly acquired base modification next to the anticodon on its activity in initiation. J Bacteriol 177, 2858-2862.

Maxam, A. M. \& Gilbert, W. (1980). Sequencing end-labeled DNA with base-specific chemical cleavages. Methods Enzymol 65, 499-560.

McCloskey, J. A., Pamela, F. \& Crain, P. F. (1998). The RNA modification database - 1998. Nucleic Acids Res 26, 196.

Meier, F., Suter, B., Grosjean, H., Keith, G. \& Kubli, E. (1985). Queuosine modification of the wobble base in $\mathrm{tRNA}^{\mathrm{His}}$ influences 'in vivo' decoding properties. EMBO J 4, 823-827.

Messing, J. (1981). A system for shotgun DNA sequencing. Nucleic Acids Res 9, 309-321.

Morris, R. C., Brown, K. G. \& Elliot, M. S. (1999). The effects of queuosine on tRNA structure and function. J Biomol Struct Dyn $16,757-774$.

Nishimura, S. (1972). Minor components in transfer RNA: their characterization, location, and function. Prog Nucleic Acids Res Mol Biol 12, 49-85.

Nishimura, S., Shindo-Okada, N., Kasai, H., Kuchino, Y., Noguchi, S., Ligo, M. \& Hoshi, A. (1983). Characterization and analysis of oncofetal tRNA and its possible application for cancer diagnosis and therapy. Recent Results Cancer Res 84, 401-412.

Noguchi, S., Nishimura, Y., Hirota, Y. \& Nishimura, S. (1982). Isolation and characterization of an Escherichia coli mutant lacking tRNA-guanine transglycosylase. Function and biosynthesis of queuosine in the tRNA. J Biol Chem 257, 6544-6550.

Okada, N., Noguchi, S., Kasai, H., Shindo-Okada, N., Ohgi, T., Goto, T. \& Nishimura, S. (1979). Novel mechanism of posttranscriptional modification of tRNA. J Biol Chem 254, 3067-3073.

Randerath, E., Agarwal, H. P. \& Randerath, K. (1984). Specific lack of the hypermodified nucleoside, queuosine, in hepatoma mitochondrial aspartate transfer RNA and its possible biological significance. Cancer Res 44, 1167-1171.

Reed, K. C. \& Mann, D. A. (1985). Rapid transfer of DNA from agarose gels to nylon membranes. Nucleic Acids Res 13, 7207-7221.

Reuter, K., Slany, R., Ullrich, F. \& Kersten, H. (1991). Structure and organization of Escherichia coli genes involved in biosynthesis of the deazaguanine derivative queuosine, a nutrient factor for eukaryotes. J Bacteriol 173, 2256-2264.

Romier, C., Reuter, K., Suck, D. \& Ficner, R. (1996). Mutagenesis and crystallographic studies of Zymomonas mobilis tRNAguanine transglycosylase reveal aspartate 102 as the active site nucleophile. Biochemistry 35, 15734-15739.

Rozenski, J., Crain, P. F. \& McCloskey, J. A. (1999). The RNA modification database: 1999 update. Nucleic Acids Res 27, 196-197.

Sambrook, J., Fritsch, E. F. \& Maniatis, T. (1989). Molecular Cloning: a Laboratory Manual, 2nd edn. Cold Spring Harbor, NY : Cold Spring Harbor Laboratory.

Seong, B. L. \& RajBhandary, U. L. (1987). Escherichia coli formylmethionine tRNA: mutations in GGG/CCC sequence conserved in anticodon stem of initiator tRNAs affect initiation of protein synthesis and conformation of anticodon loop. Proc Natl Acad Sci U S A 84, 334-338.

Slany, R. K. \& Kersten, H. (1994). Genes, enzymes and coenzymes of queuosine biosynthesis in procaryotes. Biochimie 76, 1178-1182.

Sprinzl, M., Hartman, T., Weber, J., Blank, J. \& Zeidler, R. (1989). Sequences supplement. Nucleic Acids Res 17, r1-r172.

Studier, F. W. \& Moffatt, B. A. (1986). Use of bacteriophage T7 RNA polymerase to direct selective high-level expression of cloned genes. J Mol Biol 189, 113-130.

Thanedar, S., Kumar, N. V. \& Varshney, U. (2000). The fate of the initiator tRNAs is sensitive to the critical balance between interacting proteins. J Biol Chem 275, 20361-20367.

Thanedar, S., Dineshkumar, T. K. \& Varshney, U. (2001). The mere lack of $\mathrm{rT}$ modification in initiator tRNA does not facilitate formylation-independent initiation in Escherichia coli. J Bacteriol 183, 7397-7402.

Timms, A. R. \& Bridges, B. (1996). The tyrT locus of E. coli B. J Bacteriol 178, 2469-2470.

Varshney, U., Lee, C. P. \& RajBhandary, U. L. (1991). Direct analysis of aminoacylation levels of tRNA in vivo. J Biol Chem 266, 24712-24718.

Received 21 June 2002; revised 18 August 2002; accepted 20 August 2002. 